\title{
Investigation of phytochemical components and bioautography of Garcinia mangostana L. methanol leaf extract
}

\begin{abstract}
The aim of this work to identify chemical components of Garcinia mangostana leaves and test the antibacterial effect on Pseudomonas syringe pv. tomato and Xanthomonas oryzae pv. oryzae using bioautographic procedure. Phytochemical screening of methanolic extract showed that the leaf extract of G. mangostana rich in alkaloids, Flavonoids, Saponins, Tannins, Phenol, Terpenoids, Anthraquinone and Cardiac glycosides. A simple bioautographic procedure, involving spraying suspensions of the bacteria on thin layer chromatography (TLC) plates developed in solvents of varying polarities was used to detect the number of antibacterial compounds present in the extract. This activity was indicated by white spots against a red background on the TLC plates after spraying with 5\% TTC aqueous solution. P. syringae and X. oryzae were inhibited by the most compounds separated on the TLC plates from the extract.
\end{abstract}

Keyword: TLC; Antibacterial; Xanthomonas oryzae; Pseudomonas syringe; Phytochemical screening 